\title{
Motivar el aprendizaje utilizando nuevas tecnologías: Monitorización continua de glucosa Motivate learning using new technologies: Continuous glucose monitoring
}

\author{
E. Obrador*, J.M. Estrela, S.L. Vallés, B. Pineda y V.M. Víctor \\ elena.obrador@uv.es, jose.m.estrela@uv.es, lilian.Valles@uv.es, begona.pineda@uv.es, victor.victor@uv.es \\ Departamento de Fisiología \\ Facultad de Medicina (Universidad de Valencia) \\ Valencia, España
}

\begin{abstract}
Resumen- La innovación pretende: motivar, cambiar "el saber por saber hacer", "fomentar el uso de nuevas tecnologías", etc. Creemos que este proyecto abarca todo lo expuesto. La diabetes es la enfermedad endocrina más prevalente y la $7^{\mathrm{a}}$ causa de muerte en el mundo. Los monitores continuos de glucosa son de gran ayuda para controlar la glucemia y reducir el riesgo de patología diabética. Hemos aprovechado esta idea para motivar a los estudiantes, e involucrarlos en esta propuesta de Flipped learning que nos ha llevado a elaborar un vídeo titulado "Monitorización continua de la glucemia", en el que ellos mismos explican el funcionamiento y utilidad de este avance tecnológico. Con este proyecto hemos logrado: a) que constataran la importancia del páncreas en el control de la glucemia (al comparar los resultados obtenidos en estudiantes sanos con los de pacientes diabéticos); b) fomentar el uso de nuevas tecnologías; c) motivar y favorecer el aprendizaje autónomo y colaborativo; d) hacerlos protagonistas del vídeo y responsables de trasladar lo aprendido a sus compañeros; e) crear un material docente de calidad, útil para estudiantes, profesionales de Ciencias de la Salud y pacientes diabéticos (usuarios potenciales de estos dispositivos), disponible a través de las redes sociales.
\end{abstract}

Palabras clave: innovación educativa, motivación, aprendizaje activo, aprendizaje cooperativo, competencias educación superior, entornos virtuales aprendizaje, competencias digitales, tecnologías de la información y la comunicación (TIC)

Abstract- The innovation aims to: motivate, "turning knowledge into action", "promote the use of new technologies", develop "creative talents" and so on. We believe this project encompasses all of the above. Diabetes is the most prevalent endocrine disease and the 7 th leading cause of death in the world. Continuous glucose monitors are of great help in controlling blood glucose levels and reducing the risk of diabetic disease. We have taken advantage of this idea to motivate and engage medical students in this Flipped learning proposal that led us to create a video entitled "Continuous blood glucose monitoring", in which themselves explain the usefulness of this technological advance through social networks. With this project we achieved that students could: a) directly verify the importance of the pancreas funtion in glycemic control (comparing their results with those obteined in diabetic patients); b) approach and implement the use of new technologies for learning; c) motivate and promote autonomous and collaborative learning; d) become protagonists and responsible of transferring what they have learned to their colleagues; and e) create a quality teaching material, useful also for health sciences professionals and diabetic patients, all them potential users of these devices.

Keywords: teaching-learning innovation, motivation, active learning, cooperative learning, higher education skills, virtual learning environments, digital skills, Information and Communication Technologies (ICT)

\section{INTRODUCCIÓN}

En el modelo de enseñanza tradicional, las competencias generales y específicas de las asignaturas cursadas se adquieren mediante clases presenciales, en las que el alumno dedica la mayor parte de su tiempo a escuchar al profesor y realizar trabajos fuera del horario lectivo. Aunque este modelo ha sido adoptado por la mayoría de docentes, ya en la década de los setenta se puso en duda su utilidad y eficacia. En 2012, J. Bergmann y A. Sams desarrollaron una estrategia didáctica denominada Clase Invertida (Flipped classroom), consistente en facilitar a los alumnos los contenidos del temario (apuntes, libros artículos, vídeos, etc.) antes de la clase, favoreciendo que asistan preparados y aprovechen mejor los tiempos compartidos en el aula, dando tiempo al planteamiento de casos y/o resolución de casos en un ambiente más distendido y dinámico que facilita la participación (Bergmann, 2012). Entre las ventajas de la clase invertida:

- Confiere al alumno un papel más activo en el proceso de enseñanza aprendizaje y favorece el refuerzo de lo aprendido en clase.

- Permite que el alumno aprenda según sus necesidades gracias a que puede utilizar "a demanda" el material subido en el aula virtual.

- Potencia el compromiso con el profesor, con los compañeros, el desarrollo de habilidades de comunicación, la empatía y favorece el trabajo colaborativo con los compañeros (Costelo, 2020), estableciéndose todas estas como competencias transversales del Grado de Medicina (Ministerio de Educación y Ciencia, BOE-A-2008-2674).

- Cuando esta metodología se desarrolla en grupos pequeños (como hemos hecho nosotros), el profesorado sabe quién ha accedido y revisado la información, dispone de más tiempo y puede atender necesidades individuales del aprendizaje (Tourón 2015).

Según la pirámide del aprendizaje "enseñar es la mejor manera de aprender", estimándose que los estudiantes retienen el $90 \%$ de lo aprendido cuando se lo enseñan a otros o lo utilizan de inmediato (Miller, 1990). Es cierto que los porcentajes son discutidos (Lalley, 2007) y que debemos tener en cuenta el contexto de la experiencia, la implicación de los estudiantes y 
cómo se mide el aprendizaje. En cualquier caso, por nuestra propia experiencia como docentes, la veracidad del "principio" es innegable ya que "reforzamos nuestros conocimientos cada día que damos clase". El reto es conseguir que los/as estudiantes asuman un papel activo, se preparen, participen y colaboren ya que, de lo contrario, el intento será infructuoso.

Para motivar al estudiante de Medicina, resulta de gran utilidad todo aquello que tiene que ver con la práctica clínica y el contacto con las nuevas tecnologías. El aprendizaje activo ayuda a acabar con la enquistada y tradicional pasividad asociada a la clase presencial, generando contextos que promueven situaciones para lograr un aprendizaje profundo, en las que los estudiantes se convierten en los protagonistas, fomentándose la relación con sus compañeros/as y con el profesorado. El trabajo cooperativo fomenta el aprendizaje al integrar personalidades diversas en un grupo, con más o menos destrezas para llevar a cabo tareas que deberán superar todos (Pujolàs, 2005). Además, es fácil que se establezcan debates entre iguales, que obligarán a presentar argumentos válidos que contribuyen a afianzar los conocimientos teóricos y prácticos adquiridos. Con ello, se logran mayores grados de autonomía, responsabilidad y compromiso. En todo este proceso, el apoyo y la guía del profesor es imprescindible (Girod, 2002; Ríos, 2009), ya que una adecuada retroalimentación permite reconocer los errores, mejorar los resultados y equilibrar los esfuerzos realizados por los miembros del grupo.

El Plan de Estudios 2010 de la Facultad de Medicina define a la competencia (Durante, 2011) como el conjunto de conocimientos, habilidades, actitudes, valores y aptitudes que interrelacionados entre sí permiten tener un desempeño profesional eficiente de conformidad con el estado del arte. Las competencias involucran no sólo conocimientos y técnicas, sino también el compromiso ético y valores (Peiró i Gregori, 2012) como elementos del desempeño competente. Para ello, el proceso educativo requiere del trabajo en equipo, la colaboración del profesorado, el cumplimiento de los programas académicos, el papel proactivo del estudiante y el desarrollo de sistemas integrales de evaluación (Reyes, 2010). En la medida en que profesor/a y estudiante comprenden y utilizan las competencias para el aprendizaje, estas últimas logran coherencia y congruencia. La educación por competencias implica: a) Transitar del enfoque centrado en la enseñanza hacia el enfoque centrado en el aprendizaje, b) lograr que el alumnado se apropie del control de su proceso formativo, c) realizar trabajo directo con el/la estudiante por medio de la actividad de tutoría o de asesoría, d) lograr la congruencia de cada asignatura, materia, módulo o área con las competencias, e) aplicar una diversidad de estrategias de enseñanzaaprendizaje adecuada al programa académico, f) lograr la integración básico-clínica y teórico-práctica a lo largo del currículo, g) emplear las TIC de manera cotidiana y eficiente, h) promover el trabajo colaborativo para favorecer el enfoque constructivista, i) desarrollar sistemas integrales de evaluación que tomen en cuenta el proceso curricular, el aprendizaje, la enseñanza, la retroalimentación y la actualización, j) actualizar los contenidos y materiales educativos de manera permanente, y k) promover actividades multi- e interdisciplinarias. En definitiva, la educación por competencias facilita la integración del saber, el saber hacer y el ser, esto es, la integración del conocimiento, la habilidad, las actitudes, los valores y las aptitudes (Durante, 2011), y en muchos sentidos este ha sido nuestro objetivo en este Proyecto de Innovación Docente (PID).

La Universidad de Valencia (UV) financia proyectos PID en los que se elaboran vídeos de apoyo a la docencia. Estos vídeos se difunden "on-line" a través del canal del Servei de Formació Permanent adscrito a la plataforma YouTube y también se puede acceder a ellos mediante los enlaces publicados en los cuadernillos de prácticas y/o la plataforma Moodle. Contienen explicaciones y ejemplos que resultan de gran utilidad para mejorar la preparación y el aprovechamiento de las clases (teóricas y prácticas), la preparación del examen y la adquisición de competencias. Todo ello, por no mencionar que, en estos dos últimos cursos académicos, han llegado a sustituir a la clase presencial debido a la limitación en los aforos. Nuestro departamento imparte clases teóricas de Fisiología del sistema endocrino y la práctica de "determinación de la glucemia" en la asignatura de Fisiología Médica II de $2^{\circ}$ curso del Grado de Medicina (UV). En dicha práctica, con la ayuda de un glucómetro convencional, se determina la glucemia capilar, en ayunas, tras la ingesta y durante el ejercicio físico. Los monitores continuos de glucosa (MCG), considerados en 2018 uno de los 10 dispositivos médicos más innovadores, son mucho más útiles para hacer el seguimiento de los cambios en la glucemia durante las 24 horas del día, e imprescindibles para garantizar el correcto tratamiento del paciente diabético (Lin, 2021). La Universidad de Valencia nos concedió un PID (UVSFPIE_RMD18-954130), que nos permitió comprar varios MCG, con el objetivo de favorecer la introducción en las aulas de estas nuevas tecnologías, desarrollar técnicas de innovación educativa (Peris-Ortiz, 2014) y elaborar un vídeo en el que se explican los contenidos de la práctica y el manejo de estos dispositivos. La enseñanza virtual y las TIC han sido imprescindibles para llevar a cabo este PID.

\section{CONTEXTO}

Los/as estudiantes de ciencias experimentales suelen acudir a prácticas sin revisar los manuales y sin preparación previa. Ello conlleva a errores, a la pérdida del tiempo disponible para la experimentación y a un menor aprovechamiento de la clase, que limita la adquisición de conocimientos y habilidades, entre otros. Actualmente, los estudiantes presentan un alto grado de dominio de las tecnologías de la información y la comunicación (TIC) que ofrecen enormes posibilidades educativas (Palomo, 2006). El auge de estas tecnologías ha propiciado el desarrollo del denominado aprendizaje móvil (Trillo, 2015), que permite implementar nuevas metodologías docentes, complementarias a la tradicional, que resultan más atractivas, despiertan curiosidad y facilitan la mejora del proceso de enseñanza-aprendizaje (Peris-Ortiz, 2014). Por medio del vídeo, estudiantes y docentes pueden mejorar su preparación, evaluar casos clínicos, acercarse a trabajos científicos y de actualidad, aclarar conceptos, reforzar y aplicar conocimientos y conocer la opinión de expertos. Es una herramienta que genera discusión y reflexión, mezcla imágenes, sonido y color, lo cual resulta atractivo para los/as estudiantes, convirtiéndose en un elemento motivador (Ricardo, 2017).

El ambiente y espacio de la clase teórica limitan el aprendizaje en muchos sentidos. Cohíbe a estudiantes más introvertidos/as, el escaso tiempo disponible limita la posibilidad de plantear 
preguntas y la capacidad de respuesta del profesor/a, etc. En este sentido, los grupos de prácticas, habitualmente más reducidos, son ambientes idóneos para poner en marcha estrategias de innovación. Además, establecer contacto fuera del aula, utilizar ambientes más distendidos y/o diferentes al ambiente académico, contribuye al aprendizaje (HervásGómez, 2019). En este sentido, la mayor parte de este PID ha sido llevado a cabo en la sala de prácticas y/o fuera del aula, en un ambiente proclive a las nuevas experiencias y al aprendizaje. En lo que se refiere a la práctica de la glucemia, la MCG es una técnica muy novedosa, útil para hacer el seguimiento de los cambios durante las 24 horas del día, y para garantizar el correcto tratamiento del paciente diabético. Con la financiación del PID (UV-SFPIE_RMD18-954130) compramos 4 MCG cuyo uso nos sirvió como reclamo para motivar la participación de los estudiantes en este PID.

Objetivos:

- Motivar a los/as estudiantes en el estudio de la Fisiología, promover el aprendizaje activo recurriendo al uso de las TIC y convertir a los/as estudiantes en profesores/as que, a través del vídeo MCG, explican a sus compañeros/as los contenidos de la práctica y el funcionamiento de estos dispositivos.

- Crear un ambiente propicio que favorezca el acercamiento alumno/a-profesor/a, que contribuya a la desinhibición del estudiante, a la consulta de dudas, de curiosidades y/o inquietudes, y a que se establezcan vínculos promovedores del aprendizaje y la adquisición de competencias.

- Ayudar a la adquisición de las habilidades necesarias para el desarrollo futuro de su profesión: trabajar en grupo, conocimiento de lenguaje científico, hablar en público, etc., en definitiva, fomentar el aprendizaje por competencias asociadas a los Grados de Medicina y otras titulaciones de Ciencias de la Salud.

- Crear un vídeo educativo de calidad, de libre acceso a través del canal Youtube, que será de utilidad para estudiantes de nuestra asignatura y también para estudiantes y/o profesionales de otras disciplinas dentro del campo de Ciencias de la Salud, para pacientes diabéticos y personas de su entorno.

\section{DESCRIPCIÓN}

Ofrecimos a los/as estudiantes de los 4 grupos de la asignatura de Fisiología Médica II ( $2^{\circ}$ de Medicina), la posibilidad de hacer uso de los MCG (durante 14 días, vida útil del dispositivo) para hacer el seguimiento de sus glucemias, compartir en tiempo real sus resultados con sus compañeros/as y participar en la preparación y creación del vídeo asociado a este PID. Fueron muchos los voluntarios (más de 30), a los que proporcionamos, por correo electrónico (tiempos de pandemia), la información relativa al funcionamiento de los MCG (enlaces a página web del fabricante, trabajos de investigación, etc.) y convocamos después, fuera del horario lectivo, a una reunión en la que les explicamos con más detenimiento, en qué consistiría el proyecto, cómo colocarse el MCG y las tareas a realizar para la preparación del vídeo. Expusimos que, para participar, era imprescindible preparar previamente los contenidos teóricos y prácticos de la asignatura y utilizar recursos on-line para aprender por si mismos: el procedimiento de colocación de los sensores, su activación, la descarga y la activación de las aplicaciones para hacer lecturas con el móvil, compartir sus glucemias mediante aplicaciones móviles, y poder tener así acceso e interpretar los resultados que obtuvieran los/as estudiantes que llevaran colocado el sensor. Con estas premisas pusimos en marcha dinámicas de Flipped learning (favorecedoras del autoaprendizaje), el trabajo colaborativo y el uso de las TIC (Ricardo, 2017). Tras la reunión, otros/as estudiantes se ofrecieron a participar, incluso siendo conocedores/as de que no disponíamos de MCG para todos, lo cual puso en evidencia que habíamos conseguido despertar su interés. Sólo disponíamos de 4 sensores de glucosa, con lo que nos enfrentamos al primer problema, ¿Cómo distribuir la tarea? ¿Cómo evitar que se sintieran excluidos? A partir de aquí, la secuencia de acontecimientos fue la siguiente:

a) Para no crear diferencias entre los 4 grupos de teoría, por sorteo, asignamos un sensor a un/a estudiante de cada grupo con Smartphones compatibles con los MCG (Abbott 2018). Para evitar excluir a nadie y mantener la motivación, acordamos que los/as portadores/as del sensor, compartirían sus glucemias con el resto, recurriendo al uso de la aplicación móvil de la marca, que instalaron en sus teléfonos móviles. Gracias al uso de esta aplicación móvil, pudimos involucrar a más estudiantes en el proyecto, todos/as los participantes pudieron comprobar los cambios en la glucemia a lo largo del día y los múltiples factores que la afectan y también que no todos tenemos ni los mismos valores, ni tampoco la misma capacidad de respuesta para compensar los cambios asociados a las actividades cotidianas. La utilización de las aplicaciones móviles puso en evidencia la utilidad de las nuevas tecnologías, para que los pacientes diabéticos pueden compartir de forma instantánea sus glucemias con familiares y/o personas cercanas, siendo esto de especial interés en niños.

b) Entre los voluntarios había 2 diabéticos tipo 1, usuarios habituales de los MCG que compartieron con sus compañeros/as sus conocimientos y su experiencia de vida con la diabetes, lo cual fomentó la empatía con pacientes afectados (desarrollo de lo que se conocen como competencias ocultas del Grado de Medicina). Su participación nos permitió hacer un estudio comparativo entre las glucemias de personas sanas y pacientes diabéticos, poniéndose en evidencia la importancia de los mecanismos fisiológicos de control de la glucemia. Su colaboración fue muy útil también para favorecer el trabajo colaborativo (Hadwin,2017), el aprendizaje mediante casos clínicos y la educación en valores (Peiró i Gregori, 2012) al tener la gentileza de compartir con sus compañeros/as sus datos médicos y sus conocimientos sobre el tema.

c) Reunimos de nuevo a los voluntarios, que explicaron lo que habían aprendido en su trabajo de búsqueda a través de internet, poniendo en evidencia su interés y el trabajo en grupo. Les proporcionamos los MCG y los glucómetros, con los que ensayamos lo que debían hacer (colocación y activación de los sensores, determinación de la glucemia con un glucómetro tradicional, compartir los datos utilizando el Smartphone, etc.) para estar preparados y llevar a cabo la grabación del vídeo en el laboratorio.

d) El día de la grabación en el laboratorio (3 de noviembre del 2020), los/as voluntarios/as explicaron frente a la cámara, como determinar la glucemia con el glucómetro tradicional y el procedimiento de colocación y uso de los MCG. A partir de ese momento y durante 15 días, todos tuvieron información 
instantánea de las glucemias de sus compañeros/as. Comprobamos que habíamos motivado y despertado el interés del alumnado, porque a diario nos planteaban dudas, curiosidades, ideas,... lo cual contribuyó a favorecer el acercamiento alumno/a-profesor/a. Aprovechamos el feed-back $\mathrm{y}$ el trabajo en equipo, para recomendarles que, fueran ellos mismos, los que utilizaran lo aprendido en Fisiología para interpretar los resultados, y justificar cómo afectan a la glucemia y al sistema endocrino, los cambios en la alimentación, la sobrecarga oral con glucosa, la práctica del ejercicio, las situaciones de estrés, los cambios en el ciclo ovárico, etc. Con todo ello, se fomentó el aprendizaje activo y el desarrollo de técnicas de innovación acordes a las propuestas del Espacio Europeo de Educación Superior (Tejada, 2006).

e) Una vez obtenidos los resultados, con nuestra ayuda, los/as estudiantes se encargaron de preparar la presentación en powerpoint que, posteriormente, fue utilizada en grabación y edición del vídeo titulado "Monitorización continua de la glucemia", en el que los/las estudiantes explican: el control hormonal de la glucemia, el manejo de glucómetros capilares y/o MCG, la interpretación de sus resultados, el uso de informes AGP (perfil ambulatorio de glucosa) y las ventajas e inconvenientes del uso de cada uno de estos dispositivos. De este modo, utilizamos el Flipped learning y las TIC, para favorecer la adquisición de competencias horizontales y transversales del grado de Medicina (Ministerio de Educación y Ciencia, BOE-A-20082674), como son: "Comprender y reconocer la estructura y función normal del cuerpo humano, a nivel molecular, celular, tisular, orgánico y de sistemas, en las distintas etapas de la vida y en los dos sexos, la utilización adecuada del lenguaje científico, transmitir información, ideas, problemas y soluciones a un público tanto especializado como no especializado, comprender y reconocer los efectos del crecimiento, el desarrollo y el envejecimiento sobre el individuo y su entorno social, etc.”. En principio, la segunda parte de la grabación y montaje del vídeo debía haberse llevado a cabo en diciembre, pero debido a la pandemia, a problemas administrativos (baja laboral y rescisión del contrato del técnico) y a la imposibilidad de contar con los estudiantes durante el mes de enero (por exámenes), todo se retrasó mucho más de lo esperado, propiciando la desvinculación de algunos alumnos/as, lo cual es fácilmente comprensible si tenemos en cuenta que, una vez aprobada la asignatura, se centran en las actividades del nuevo cuatrimestre.

f) Tan pronto se nos asignó un nuevo técnico de grabación, contactamos con voluntarios/as (ahora unos 14) para ver quienes seguían interesados y nos iban a ayudar a finalizar el proyecto, llevando a cabo la grabación del vídeo en el estudio. El nuevo técnico nos indicó que no debían ser más de 4-5 personas las que aparecieran en el vídeo. Los propios estudiantes fueron los que llevaron a cabo el proceso de selección, en función de su disponibilidad horaria y la del técnico de grabación. El resto de estudiantes han permanecido vinculados al proyecto hasta el final, ayudándonos en la preparación de la presentación power point y del vídeo.

\section{Resultados}

A. Vídeo: "Monitorización continua de la glucemia"

De acceso libre a través del canal Youtube del Servei de Formació Permanent (UV). En la figura 1 aparece el enlace de acceso al vídeo, se describen sus contenidos y aparecen los/as participantes.

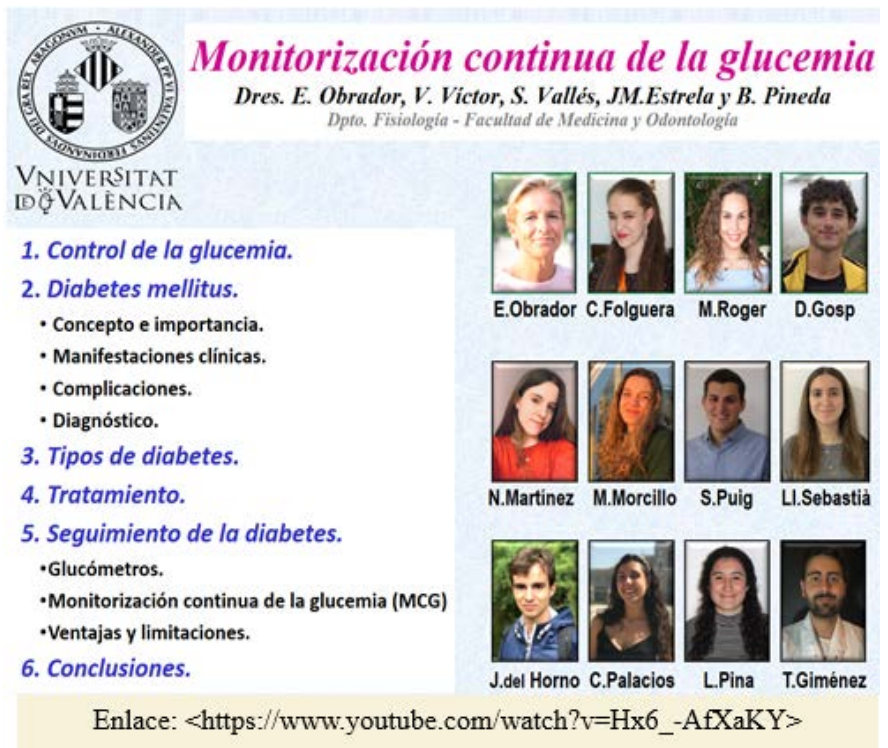

Figura 1. Portada del vídeo con fotografía de los participantes.

\section{B. Motivar y favorecer el aprendizaje activo.}

Hemos implementado estrategias de aprendizaje activo, que más allá de ayudar a superar el examen de evaluación, han ayudado a acercar al alumnado los últimos avances tecnológicos, a fomentar el trabajo colaborativo (Girod, 2002; Hadwin, 2017), al conocimiento y uso del lenguaje científico, a la utilización de las TIC (Palomo, 2006; Ricardo, 2017) y, desde luego, a estrechar vínculos alumno/a-profesor/a, favoreciendo con ello la adquisición de competencias esenciales en la formación de un médico (Ministerio de Educación y ciencia, BOE-A-2008-2674; Durante, 2011; Reyes, 2010).

Los/as voluntarios/as que se mantuvieron vinculados al PID hasta el final contestaron a una encuesta anónima, y estas fueron las opiniones que recogimos:

- El 100\% manifestaron que: a) su participación en el PID les había ayudado en la adquisición de competencias de la asignatura, b) había ayudado a establecer vínculos más estrechos con el profesorado, c) recomendaría participar a sus compañeros, d) vídeo es de utilidad para futuros alumnos y/o compañeros de carrera y para pacientes diabéticos.

- El 85,7\% opina que lo aprendido les será de utilidad en otras asignaturas y en su actividad profesional. El 14,3\% restante manifiesta que no lo sabe.

- El 92,9\% manifiesta que: a) se ha fomentado el uso de las nuevas tecnologías, b) el trabajo colaborativo y c) volvería a presentarse como voluntario/a. El resto no contesta.

- El 71,4\% opina que con su participación se ha ayudado a los compañeros de clase, y el $28,6 \%$ no lo sabe.

\section{Futuros resultados.}

En los próximos cursos utilizaremos el vídeo para desarrollar estrategias de Flipped classroom. Para valorar la utilidad del vídeo, plantearemos encuestas de opinión y comprobaremos si, con la disponibilidad de este material, se mejora la preparación de la práctica y los resultados de la evaluación. Más allá del ámbito educativo, se evaluará la utilidad del vídeo, revisando el número de visitas en Youtube y las opiniones de usuarios/as. 


\section{CONCLUSIONES}

Gracias a este proyecto:

- Los/as estudiantes han utilizado MCG de última generación, revisar e interpretar sus informes, y comprobar directamente los cambios en la glucemia asociados a las actividades cotidianas, trasladando a su día a día lo aprendido en clase. Hemos cambiado "el saber, por el saber hacer".

- Hemos favorecido el acercamiento alumno/a-profesor/a y el establecimiento de vínculos que facilitan el aprendizaje activo.

- Los/as estudiantes se han convertido en profesores/as responsables de crear un material educativo de calidad. Hemos utilizado las TIC para facilitar el aprendizaje y hemos implementado estrategias de Flipped learning con las que hemos conseguido despertar el interés, la motivación y favorecer el aprendizaje activo y la adquisición de competencias, horizontales y transversales del Grado de Medicina.

- El vídeo de MCG ayudará a futuros estudiantes a preparar y aprovechar mejor la práctica, y facilitará la adquisición de competencias. El libre acceso a través de las redes beneficiará también a estudiantes matriculados en otros cursos y/o Grados de Ciencias de la salud, profesionales sanitarios y pacientes diabéticos, usuarios potenciales de estos dispositivos.

\section{REFERENCIAS}

Abbott (2018). Continuous Glucose Monitoring System. Recuperado 27 de agosto, 2021, de Freestyle Libre: https://www.freestylelibre.us/.

Bergmann, J. \& Sams, A. (2012). Flip Your Classroom: Reach Every Student in Every Class Every Day. Virginia: ASCD.

Costelo, G.J. (2020). The Teaching of Design and Innovation. Principles and Practices. Cham, Suiza: Editorial Springer Nature.

Durante, I., Martínez A., Morales S., Lozano J.R. \& Sánchez M. (2011). Educación por competencias: de estudiante a médico. Revista de la Facultad de Medicina 54 (6):42-50.

Girod, G.R. (2002). Connecting Teaching and Learning. A Handbook for teacher educators on Teacher Work Sample Methodology. Washington: Editorial AACTE-ERIC.

Hadwin, A.F., Järvelä, S. \& Miller, M. (2017). Self-regulation, co-regulation and shared regulation in collaborative learning environments. En Schunk, D.H. \& Greene, J.A. (Eds). Handbook of Self-Regulation of Learning and Performance. New York: Routledge.

Hervás-Gómez, C., Vázquez-Cano, E., Fernández Batanero J.M. \& López-Meneses E. (2019). Innovación e investigación sobre el aprendizaje ubicuo y móvil en la Educación Superior. Barcelona. Ediciones Octaedro S.L.
Lalley, J. \& Miller, R. (2007). The Learning Pyramid: Does It Point Teachers in the Right Direction? Revista Education, 64-79.

Lin, R., Brown, F., James, S., Jones, J. \& Ekinci, E. (2021). Continuous glucose monitoring: A review of the evidence in type 1 and 2 diabetes mellitus. Diabetic Medicine, 38(5): e14528. doi: 10.1111/dme.14528.

Miller G.E. (1990). The assessment of clinical skills/ competence/performance. Academic Medicine 65 (9), 63 7.

Ministerio de Educación y Ciencia. Requisitos para la verificación de los títulos universitarios oficiales que habiliten para el ejercicio de la profesión de Médico. BOE, 15 de febrero de 2008, núm. 40, p.8351 a 8355. $<$ https://www.boe.es/eli/es/o/2008/02/13/eci332>.

Palomo R., Ruiz J. \& Sánchez, J. (2006). Las TIC como agentes de innovación educativa. Andalucía: Junta de Andalucía. Consejería de Educación Dirección General de Innovación Educativa y Formación del Profesorado.

Peiró i Gregori, S. (2012). Innovaciones sobre la formación inicial de profesores con relación a la educación en valores. Recuperado agosto 27, 2021, de https://www.redalyc.org/articulo.oa? $\mathrm{id}=217024398005$

Peris-Ortiz, M., Garrigós-Simón F.J. \& Gil Pechuán J. (2014). Innovation and Teaching Technologies. New Directions in Research, Practice and Policy. Suiza: Editorial Springer.

Pujolàs Maset, P. (2005). El cómo, el porqué y el para qué del aprendizaje cooperativo. Cuadernos de Pedagogía, 345, 51-54.

Reyes E. (2010). Enfoque curricular basado en competencias en la educación médica. Comunidad y Salud, 8(1), 58-64.

Ricardo Bareto, C. \& Iriarte Diazgranados F. (2017). Las Tic en Educación Superior: Experiencias De Innovación. Colombia: Editorial Universidad Del Norte.

Ríos Muñoz, D. (2009). Rasgos de personalidad de profesores innovadores: autonomía, persistencia y orden. Revista Latinoamericana de Estudios Educativos, 31 (2), 95-112.

Tejada Artigas, C.M. et al. (2006). El diseño del plan docente en Información y Documentación acorde con el Espacio Europeo de Educación Superior: un enfoque por competencias. [Documento en PDF]. https://eprints.ucm.es/id/eprint/6005/1/MANUAL.pdf.

Tourón, J. \& Santiago, R. (2015). El modelo Flipped Learning y el desarrollo del talento en la escuela. Educación, 368, 196-231.

Trillo, M.P. (2015). Principios pedagógicos del aprendizaje ubicuo. Dispositivos digitales móviles en Educación. El aprendizaje ubicuo, 39-48. 